\title{
Characterization of Breast Lesions: Comparison of Digital Breast Tomosynthesis and Ultrasonography
}

\author{
Sun Ah Kim, $M D^{1}$, Jung Min Chang, $M D^{2}$, Nariya Cho, $M D^{2}$, Ann Yi, $M D^{3}$, Woo Kyung Moon, $M D^{2}$
}

${ }^{1}$ Department of Radiology, Human Medical Imaging \& Intervention Center, Seoul 135-120, Korea; ${ }^{2}$ Department of Radiology, Seoul National University Hospital, Seoul 110-744, Korea; ${ }^{3}$ Department of Radiology, Seoul National University Hospital Healthcare System Gangnam Center, Seoul 135-984, Korea

Objective: To compare the diagnostic performance of digital breast tomosynthesis (DBT) and conventional breast ultrasound (US) to characterize breast lesions as benign or malignant.

Materials and Methods: A total of 332 women, presenting for screening examinations or for breast biopsy between March and June 2012 were recruited to undergo digital mammography (DM), DBT, and breast US examination. Among them, 113 patients with 119 breast lesions depicted on DM were finally included. Three blinded radiologists performed an enriched reader study and reviewed the DBT and US images. Each reader analyzed the lesions in random order, assigned Breast Imaging Reporting and Data System (BI-RADS) descriptors, rated the images for the likelihood of malignancy (\%) and made a BI-RADS final assessment. Diagnostic accuracy, as assessed by the area under the receiver operating characteristic curve, sensitivity, and specificity of DBT and US were compared.

Results: Among the 119 breast lesions depicted on DM, 75 were malignant and the remaining 44 were benign. The average diagnostic performance for characterizing breast lesions as benign or malignant in terms of area under the curve was 0.899 for DBT and 0.914 for US ( $p=0.394)$. Mean sensitivity ( $97.3 \%$ vs. $98.7 \%, p=0.508)$ and specificity $(44.7 \%$ vs. $39.4 \%, p=$ 0.360 ) were also not significantly different.

Conclusion: Digital breast tomosynthesis may provide similar reader lesion characterization performance to that of US for breast lesions depicted on DM.

Index terms: Digital breast tomosynthesis; Breast US; Diagnostic performance

\section{INTRODUCTION}

Mammography is the only screening test that has been

Received August 1, 2014; accepted after revision December 26, 2014.

This research was supported by Seoul National University Hospital Research Fund (grant 06-2012-0780).

Corresponding author: Jung Min Chang, MD, Department of Radiology, Seoul National University Hospital, 101 Daehak-ro, Jongno-gu, Seoul 110-744, Korea.

- Tel: (822) 2072-2518 - Fax: (822) 743-6385

- E-mail: imchangjm@gmail.com

This is an Open Access article distributed under the terms of the Creative Commons Attribution Non-Commercial License (http://creativecommons.org/licenses/by-nc/3.0) which permits unrestricted non-commercial use, distribution, and reproduction in any medium, provided the original work is properly cited. shown to reduce breast cancer-related mortality, and the overall sensitivity of mammography is $70-90 \%$ (1-4). However, despite its clearly documented benefits, digital mammography (DM) does not eliminate the fundamental limitation of detecting noncalcified breast cancers, which are obscured by surrounding and overlying dense parenchyma, and sensitivity is as low as $30-48 \%$ in women with dense breast tissue $(5,6)$.

Recent technical developments in DM have enabled the introduction of digital breast tomosynthesis (DBT) as a clinical imaging modality. DBT allows reconstruction of planes from breast tissue volume viewed in sequential sections through the breast and potentially overcomes this inherent limitation of DM caused by overlapping normal and pathological tissue during standard two-dimensional 
(2D) projections (7-15). DBT offers potential advantages for evaluating masses, areas of architectural distortion, and asymmetries compared with those of conventional $2 \mathrm{D}$ mammographic images (15). In diagnostic settings, DBT improves work-up efficiency and the selection of patients recommended for biopsy, thereby reducing associated costs and additional imaging studies including additional mammographic views and unnecessary biopsies (16).

As an another effective diagnostic tool to evaluate specific areas of abnormality discovered on a clinical examination or on mammography, breast ultrasonography (US) has long been used as a popular diagnostic tool, as it is widely available and well-tolerated by patients (17-22). However, the diagnostic performance of DBT compared with that of US to characterize breast lesions has not been well reported.

Therefore, in the present study, we compared the diagnostic performance of DBT and US to distinguish benign from malignant breast lesions depicted on DM in a cancerenriched population.

\section{MATERIALS AND METHODS}

\section{Study Population}

The Institutional Review Board of our hospital approved this retrospective study, and written informed consent to acquire DBT images was obtained from all participants. Between March and June 2012, full-field DM with twoview DBT, i.e., craniocaudal (CC) and mediolateral oblique (MLO) (simultaneous acquisition of DM and DBT images, COMBO procedure, Selenia Dimensions System; Hologic, Bedford, MA, USA) and US were performed in 332 patients for both diagnostic and screening purposes. A radiologist, with 3 years of experience in breast imaging, who had knowledge of the clinical data but did not take part in the reader study, selected the study population. of the 332 consecutive patients who underwent DBT and US, 164 who presented with at least one breast mass, focal asymmetry, or architectural distortion on DM were identified. Patients who had only microcalcifications without a mass,

asymmetry, or architectural distortion were not included in this study. Among the 164 patients, 51 were excluded due to the following reasons: 1 ) indeterminate correlation of the mass location on DM and US $(n=11) ; 2)$ no biopsy data or follow-up data of $<24$ months $(n=34)$; and 3 ) patients who had undergone neoadjuvant chemotherapy before DBT, DM, and US imaging $(n=6)$. Finally, 113 consecutive patients (age range, 29-78 years; mean age, 49.6 years) with 119 breast lesions constituted our study population. Among them, 107 patients had one lesion, two patients had one lesion in each breast, and the other four patients had two lesions in one breast.

Of these 113 women, 43 (38.1\%) were asymptomatic, and $68(60.2 \%)$ presented with palpable abnormalities, one $(0.9 \%)$ had pain, and one $(0.9 \%)$ had nipple discharge. Mammographic breast density was recorded according to the American College of Radiology Breast Imaging Reporting and Data System (BI-RADS) criteria (23). Breast tissue densities on DM were as follows: pattern 1, eight patients; pattern 2, 33 patients; pattern 3, 57 patients; and pattern 4, 21 patients. Lesions were observed as a mass in 88 cases $(73.9 \%)$, as a mass with microcalcifications in 19 cases $(16.0 \%)$, as an architectural distortion in one case $(0.8 \%)$, and as focal asymmetry in 11 cases $(9.2 \%)$ on DM.

This case selection process produced a cancer-enriched study population, of which $63 \%$ (75 of 119) of the masses were malignant and $27 \%$ (44 of 119) were benign. A total of 111 lesions were confirmed through US-guided core needle biopsy (14-gauge automated gun or 11-gauge vacuumassisted) $(n=22)$ or surgical excision $(n=89)$. Total mastectomy or breast-conserving surgery was performed for all breast cancers, and lesions with benign biopsy results remained unchanged during the follow-up period (mean \pm standard deviation $9.7 \pm 1.0$ months, range 9-12 months). Eight benign cases were assessed as benign from clinical and radiological findings and were stable on recent 2-year follow-up mammography or US (mean follow-up duration, 29.6 months; range, 24-52 months).

\section{Image Acquisition}

\section{Digital Breast Tomosynthesis}

Digital breast tomosynthesis and DM were performed by one trained and dedicated technologist using a commercially available device (Selenia Dimensions System; Hologic, Bedford, MA, USA). The device consisted of a custom-designed high-power (mA) tungsten (W) anode $X$-ray tube and rhodium, silver, and aluminum $X$-ray filters. Different filters in DBT and DM imaging modes produce optimal X-ray spectra (20-49 kVp) based on the thickness/ composition of the breast using automatic exposure control. The image receptor was a 70- $\mu \mathrm{m}$ pixel pitch selenium direct capture detector. The $\mathrm{X}$-ray tube moved over a $15^{\circ}$ arc while the breast was compressed, taking a series of ultra-low dose 
mammograms. The projections were combined to create a full three dimensional-image set of the breast with 1-mm slices through the breast (20).

\section{Ultrasonography}

All US images were obtained with knowledge of the mammographic findings by one of eight breast radiologists with 3-14 years of experience in breast US on the same day as DBT and DM imaging. Bilateral whole breast US was performed with patients in the supine position using an EUB8500 scanner (Hitachi Medical, Tokyo, Japan) with a 14-6MHz linear transducer or an Aixplorer scanner (SuperSonic Imagine, Aix en Provence, France) equipped with a 15-4$\mathrm{MHz}$ linear array transducer. The scanning protocol included gray-scale images of breast mass lesions obtained in at least two orthogonal planes (transverse/longitudinal or radial/antiradial imaging). Two to four representative B-mode US images of the breast masses per case were saved as Digital Imaging and Communications in Medicine (DICOM) files from a picture archiving and communications system (PACS) by one radiologist. Doppler or elastography images were not included in the image review. All image files were masked and randomized by a research assistant.

\section{Observer Performance Study}

A database with anonymized images corresponding to patients who underwent both DBT and US was created in random order. Three readers (with 14, and 8 , and 8 years of experience in mammography and breast US, respectively) from two academic institutions were involved in the reader study. All readers had 6 months of experience in DBT, and two had participated in a previous reader study that included a different study cohort. Before the reader study, the three readers had a DBT image training period with training sets including 50 cases ( 32 cancers and 18 benign lesions) that were acquired at our institution for other research purposes and were not included in our study. The readers were shown the DBT stacks of training sets with information about tumor location before gaining access to the pathological results.

After the training period, the three radiologists independently evaluated the DBT and US images that were previously anonymized and randomized in a separate session. In the first session, the readers evaluated the DBT images of 119 breast lesions, and they analyzed US images on a different day in the second session. There was no scheduled interval between the reading sessions because we presumed that there would be no learning effect bias between the US and DBT datasets. The readers were blinded to all clinical data and DM findings. However, information on lesion location was provided to the readers because the aim of this study was to investigate reader performance for characterizing breast lesions with positive findings on DM rather than the detection of lesions.

The DBT datasets were displayed using two highresolution monitors (5 mega-pixels). The workstation allowed viewing the DBT images of both breasts, one after the other (CC views and then MLO views of both breasts together). The radiologists were blinded to the mammographic views at that moment. Each reader carefully reviewed all images for each lesion and assigned BI-RADS descriptors for the lesions on mammography (i.e., mass shape, margin, density, microcalcifications, and architectural distortion). A final BI-RADS assessment category and the probability of malignancy in a percentage (\%) were also provided $(23,24)$. In addition, the readers were asked to choose recommendations for follow-up (for lesions that were either benign or probably benign) or biopsy (for lesions that were either suspicious or highly suggestive of malignancy). Each observer was provided a form containing lesion descriptors, the BI-RADS final assessment categories, the predicted likelihood of malignancy (\%), and further management plans, and they were instructed to select the most appropriate descriptors for each lesion.

Ultrasonography scans saved as DICOM files were retrieved and viewed at a PACS workstation (Marosis, Marotech, Seoul, Korea). Representative B-mode US images of mass lesions were also evaluated for their morphological characteristics (using descriptors according to the BI-RADS lexicon i.e., mass shape, margin, orientation, boundary, posterior acoustic features, and echo pattern), final BIRADS category (24), likelihood of malignancy (\%), and the management plan.

\section{Statistical Analysis}

Analyses were performed on data obtained from the matched image sets $(n=119)$. Sensitivity and specificity of DBT and US were calculated using the binary management decision of whether each reader recommended biopsy, and they were compared using the McNemar test. A receiver operating characteristic (ROC) curve analysis was performed to evaluate the diagnostic performance of the two modalities (DBT and US) for distinguishing between benign and malignant breast lesions. The area under the 
ROC curve (AUC) was calculated using the likelihood of malignancy and was compared to characterize the breast mass lesions on DBT and US. In addition, sensitivities, specificities, and positive predictive values (PPVs) for all readers were calculated using the cut-off points yielding the maximum AUC value, and several different cut-off points were calculated. Finally, interobserver agreement among the three radiologists was estimated using $\mathrm{K}$ statistics with regard to the US/DBT findings and BI-RADS final assessment categories. A value of $\geq 0.20$ was considered slight agreement; $0.21-0.40$, fair agreement; $0.41-0.60$, moderate agreement; $0.61-0.80$, substantial agreement; and 0.811.00 , almost perfect agreement. A two-sided significance level of $5 \%$ was used for all analyses. Statistical analyses were performed using SPSS ver. 19.0 (SPSS Inc., Chicago, IL, USA) and MedCalc (MedCalc, Mariakerke, Belgium) software.

\section{RESULTS}

\section{Characteristics of the Breast Masses}

Of the 119 breast lesions, surgical excision was performed on 89 lesions. Mastectomy $(n=18)$ or breast-conserving surgery $(n=53)$ were performed for 71 patients with 75 malignant masses on biopsy.

Malignant lesions $(n=75)$ included infiltrating ductal carcinoma ( $n=67)$, ductal carcinoma in situ $(n=5)$, invasive lobular carcinoma $(n=2)$, and invasive papillary carcinoma $(n=1)$. Benign lesions $(n=44)$ included fibroadenoma $(n=22)$, fibrocystic changes $(n=4)$, benign phyllodes $(n=2)$, ductal epithelial hyperplasia $(n=1)$, granulomatous mastitis $(n=1)$, radial scar $(n=$ $1)$, sclerosingadenosis $(n=2)$, nodular adenosis $(n=1)$, galactocele $(n=1)$, and an intramammary lymph node $(n=1)$. The remaining eight benign cases were assessed as benign over $>2$ years of clinical and radiological follow-up findings.

\section{Diagnostic Reader Performance}

Table 1 reports the AUC values and the sensitivity and specificity values for each reader. The ROC analysis indicates that the AUC values estimated using the likelihood of malignancy (\%) by DBT and US were 0.896 and $0.935(p=$ $0.150)$ for reader $1,0.917$ and $0.905(p=0.648)$ for reader 2 , and 0.932 and $0.911(p=0.480)$ for reader 3, respectively. The overall AUC values for the three readers were 0.899 for conventional DBT and 0.914 for US $(p=0.394)$. The optimal cut-off point for both DBT and US that provided the maximal sum of sensitivity and specificity was between BI-RADS final assessments of 3 and 4A. Using this cut-off point, DBT and US sensitivities were $98.7 \%$ (74 of 75) and $100 \%$ (75 of 75) for reader 1 ( $p=1.000$ ), 100\% (75 of 75) and $98.7 \%(74$ of 75$)$ for reader $2(p=1.000)$, and $93.3 \%$ (70 of 75 ) and $97.3 \%$ (73 of 75) for reader $3(p=0.453)$, respectively. The mean sensitivities for the three readers were not different between DBT $(97.3 \%)$ and US (98.7\%) $(p=0.508$ ). DBT and US specificities were $36.4 \%$ (16 of 44 ) and $43.2 \%$ (19 of 44$)$ for reader $1(p=0.581), 25.0 \%$ (11 of 44) and 31.8\% (14 of 44) for reader $2(p=0.549)$, and $72.7 \%$ (32 of 44$)$ and $43.2 \%$ (19 of 44 ) for reader 3 $(p=0.004)$, respectively. A significantly higher specificity was found for DBT by reader 3; however, no significant differences were observed between DBT and US for the other two readers. The mean specificities for the three readers were not different between DBT $(44.7 \%)$ and US (39.4\%) ( $p$ $=0.360$ ).

Regarding PPVs, among lesions recommended for either short-interval follow-up or biopsy (BI-RADS categories 3-5) on DBT, $67.3 \%$ were confirmed as malignant, whereas $66.2 \%$ of lesions that were assigned BI-RADS categories 3-5 were malignant on US. A mean of $74.9 \%$ of the lesions that were recommended for biopsy by DBT (BI-RADS category 4 or 5) were malignant, and $75.3 \%$ of the lesions that were assigned BI-RADS category 4 or 5 using US were confirmed to be malignant. In addition, all lesions that were assigned BI-RADS category 5 were malignant, regardless of modality.

Table 1. Performance of Three Readers for Characterization of Breast Lesions at DBT and US

\begin{tabular}{|c|c|c|c|c|c|c|c|c|c|c|}
\hline & \multicolumn{4}{|c|}{ Az Value } & \multicolumn{3}{|c|}{ Sensitivity* (\%) } & \multicolumn{3}{|c|}{ Specificity* (\%) } \\
\hline & DBT & US & $95 \% \mathrm{CI}^{\dagger}$ & $P$ & DBT & US & $P$ & DBT & US & $P$ \\
\hline Reader 1 & 0.896 & 0.935 & $-0.014,0.094$ & 0.150 & $98.7(74 / 75)$ & $100(75 / 75)$ & 1.000 & $36.4(16 / 44)$ & $43.2(19 / 44)$ & 0.581 \\
\hline Reader 2 & 0.917 & 0.905 & $-0.038,0.062$ & 0.648 & $100(75 / 75)$ & $98.7(74 / 75)$ & 1.000 & $25.0(11 / 44)$ & $31.8(14 / 44)$ & 0.549 \\
\hline Reader 3 & 0.932 & 0.911 & $-0.036,0.077$ & 0.480 & $93.3(70 / 75)$ & $97.3(73 / 75)$ & 0.453 & $72.7(32 / 44)$ & $43.2(19 / 44)$ & 0.004 \\
\hline Overall & 0.899 & 0.914 & $-0.019,0.049$ & 0.394 & 97.3 & 98.7 & 0.508 & 44.7 & 39.4 & 0.360 \\
\hline
\end{tabular}

Note. - *Data in parentheses were used to calculated percentages. Optimal cut-off, between Breast Imaging Reporting and Data System category 3 and $4 \mathrm{~A}$ was used to yield maximal sum of sensitivity and specificity in both modality, ${ }^{\dagger} \mathrm{CI}=$ confidence interval for difference between DBT and US. DBT = digital breast tomosynthesis, US = ultrasound 


\section{Management Decisions}

Breast Imaging Reporting and Data System descriptors for lesions on DBT using the mammography (MG) BIRADS lexicon (i.e., mass shape, margin, density, microcalcifications, and architectural distortion) and on US (i.e., mass shape, margin, orientation, boundary, posterior acoustic features, and echo pattern) are summarized in Tables 2 and 3. Significant differences were observed in the distribution of the lesion descriptors on US and DBT.

Table 4 shows the management decisions of the three readers for the 119 breast masses based on DBT and US imaging. Although significant differences were detected on both US and DBT overall, the management plans were discordant between DBT and US in 15 cases (12.6\%, $15 / 119)$ for reader 1 , in 12 cases $(10.1 \%, 12 / 119)$ for reader 2 , and in 26 cases $(21.8 \%, 26 / 119)$ for reader 3. Five cancers that were determined to be benign on DBT were correctly categorized as malignant on US by at least one of the readers (one cancer by two readers and four cancers by one reader). All of these lesions showed indistinct margins on DBT images (Fig. 1). In contrast, two cancers categorized as benign on US images were correctly categorized as malignant on DBT images by at least one of the three readers (Fig. 2). These cancers were described

Table 2. DBT Lesion Characteristics

\begin{tabular}{|c|c|c|c|}
\hline Lesion Characteristics on US & Benign & Malignancy & $P$ \\
\hline Shape & & & $<0.0001$ \\
\hline Oval & 31 & 24 & \\
\hline Round & 56 & 23 & \\
\hline Lobular & 23 & 14 & \\
\hline Irregular & 22 & 164 & \\
\hline Margin & & & $<0.0001$ \\
\hline Circumscribed & 85 & 17 & \\
\hline Microlobulated & 9 & 12 & \\
\hline Obscured & 8 & 14 & \\
\hline Indistinct & 23 & 73 & \\
\hline Spiculated & 7 & 109 & \\
\hline Density & & & $<0.0001$ \\
\hline High & 43 & 147 & \\
\hline Equal & 83 & 76 & \\
\hline Low & 3 & 2 & \\
\hline Fat containing & 3 & 0 & \\
\hline Microcalcifications & & & $<0.0001$ \\
\hline No & 121 & 120 & \\
\hline Yes & 11 & 105 & \\
\hline Architectural distortion & & & $<0.0001$ \\
\hline No & 123 & 100 & \\
\hline Yes & 9 & 125 & \\
\hline
\end{tabular}

Note. - DBT = digital breast tomosynthesis, US = ultrasound as oval circumscribed hypoechoic non-calcified masses by all readers using the US images. However, one of these masses showed a spiculated margin, and another lesion showed microcalcifications on DBT. Table 5 summarizes the malignant cases considered benign using either DBT or US by at least one reader. No cancer was considered benign on both DBT and US. Among the 44 benign masses, all three readers recommended follow-up rather than biopsy with DBT in eight cases (Fig. 3) and with US in eight cases (Fig. 4); however, $20.5-59.1 \%$ of benign lesions were recommended for biopsy using both imaging tests by the three readers.

\section{Interobserver Agreement}

Interobserver agreement among the three readers in terms of the DBT findings was substantial for calcification (0.673) and architectural distortion (0.738), moderate for mass shape (0.427) and mass margin (0.546), and fair for density (0.234). Interobserver agreement for the US findings among the three readers was substantial for shape (0.636) and the

\section{Table 3. US Lesion Characteristics}

\begin{tabular}{|c|c|c|c|}
\hline Lesion Characteristics on US & Benign & Malignancy & $P$ \\
\hline Shape & & & $<0.0001$ \\
\hline Oval & 83 & 24 & \\
\hline Round & 10 & 5 & \\
\hline Irregular & 39 & 196 & \\
\hline Margin & & & $<0.0001$ \\
\hline Circumscribed & 89 & 17 & \\
\hline Indistinct & 31 & 91 & \\
\hline Angular & 3 & 45 & \\
\hline Microlobulated & 9 & 27 & \\
\hline Spiculated & 0 & 45 & \\
\hline Echogenicity & & & $<0.0001$ \\
\hline Non-hypoechoic & 50 & 22 & \\
\hline Hypoechoic & 82 & 203 & \\
\hline Orientation & & & $<0.0001$ \\
\hline Parallel & 111 & 97 & \\
\hline Not parallel & 21 & 128 & \\
\hline Lesion boundary & & & $<0.0001$ \\
\hline Abrupt interface & 125 & 110 & \\
\hline Echogenic halo & 7 & 115 & \\
\hline Posterior feature & & & $<0.0001$ \\
\hline Normal & 60 & 79 & \\
\hline Enhancement & 63 & 56 & \\
\hline Shadowing & 7 & 63 & \\
\hline Mixed & 2 & 27 & \\
\hline Microcalcifications & & & $<0.0001$ \\
\hline No & 132 & 179 & \\
\hline Yes & 0 & 46 & \\
\hline
\end{tabular}

Note. - US = ultrasound 
Table 4. Management Decision in 119 Breast Lesions at DBT and US

\begin{tabular}{|c|c|c|c|c|c|c|c|c|}
\hline \multirow[b]{2}{*}{ Plan at DBT } & \multicolumn{4}{|c|}{ Malignant Masses $(n=75)$} & \multicolumn{4}{|c|}{ Benign Masses $(n=44)$} \\
\hline & Biopsy & Biopsy & $F / U$ & $F / U$ & Biopsy & Biopsy & $F / U$ & $F / U$ \\
\hline Plan at US & Biopsy & F/U & Biopsy & $F / U$ & Biopsy & F/U & Biopsy & $F / U$ \\
\hline Reader 1 & 74 & 0 & 1 & 0 & 20 & 8 & 5 & 11 \\
\hline Reader 2 & 74 & 1 & 0 & 0 & 26 & 7 & 4 & 7 \\
\hline Reader 3 & 68 & 2 & 5 & 0 & 9 & 3 & 16 & 16 \\
\hline Total & 216 & 3 & 6 & 0 & 55 & 18 & 25 & 34 \\
\hline
\end{tabular}

Note. - Values are numbers of patient. DBT = digital breast tomosynthesis, US = ultrasound
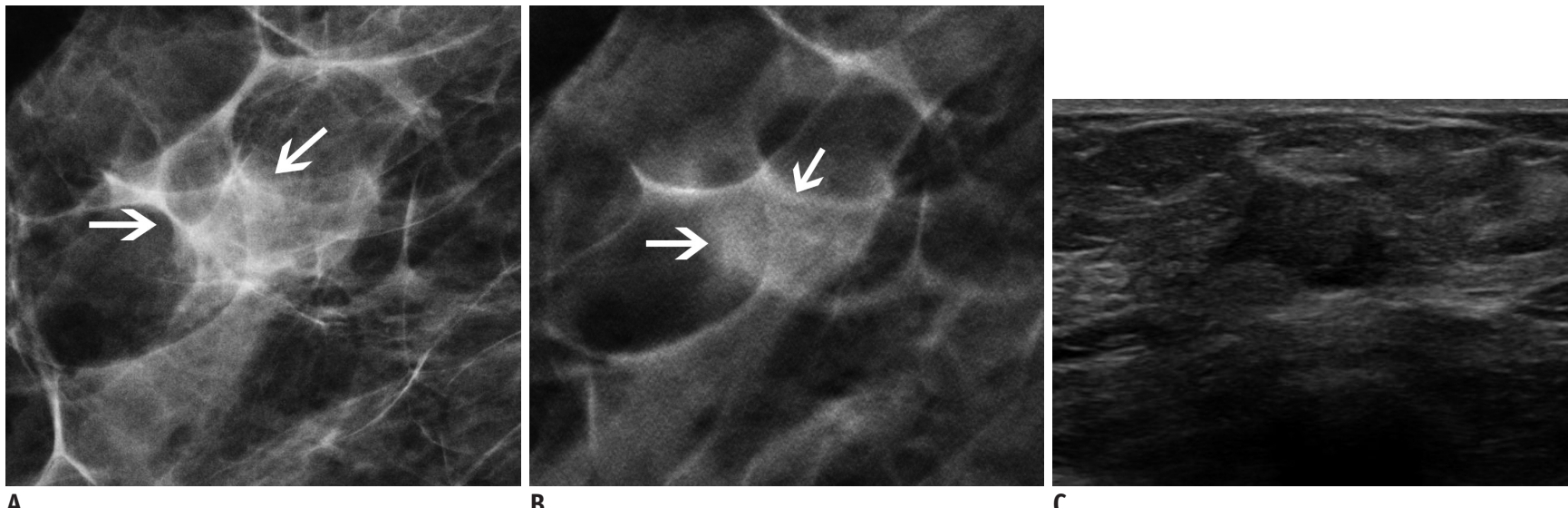

Fig. 1. 62-year-old woman with $1.2 \mathrm{~cm}$-sized invasive ductal carcinoma.

A. Craniocaudal digital mammography view. B. Craniocaudal digital breast tomosynthesis (DBT) view (1-mm section). C. Transverse

ultrasonography (US) view. Oval isodense mass (arrows) with indistinct margins is observed on DBT. This lesion appears as hypoechoic lesion with angular margins on US. Readers 1 and 3 categorized this lesion as Breast Imaging Reporting and Data System (BI-RADS) category 3, and reader 2 categorized this lesion as BI-RADS category 4 A on DBT. Readers 1 and 2 categorized this lesion as BI-RADS category 4C, and reader 3 categorized this lesion as BI-RADS category $4 \mathrm{~A}$ on US.
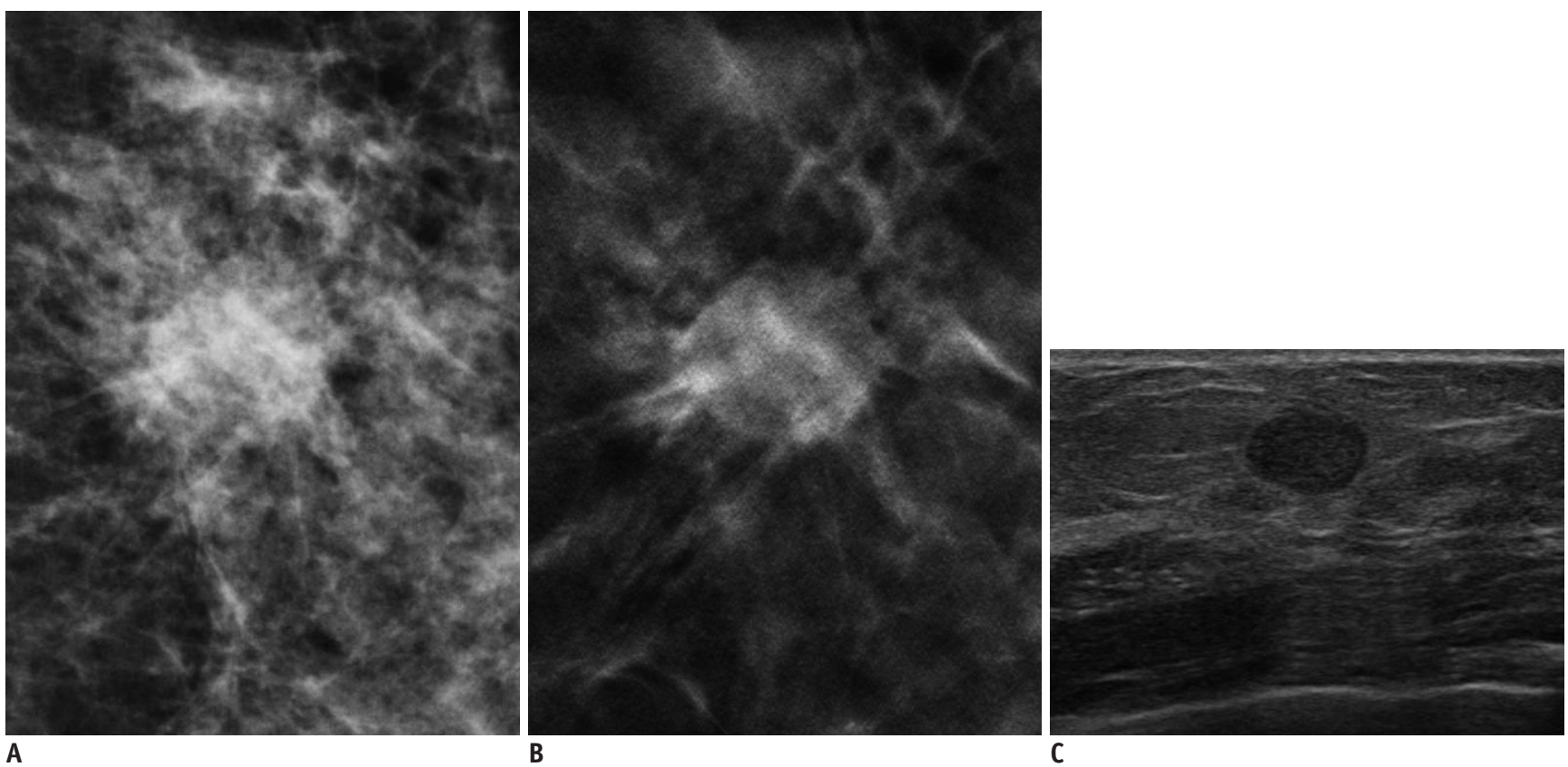

Fig. 2. 57-year-old woman with $1.2 \mathrm{~cm}$-sized invasive papillary carcinoma.

A. Craniocaudal full-field digital mammography view. B. Craniocaudal digital breast tomosynthesis (DBT) (1-mm section) view. C. Transverse ultrasonography (US) view. Mass with spiculated margin is clearly visible on DBT image. This lesion appears as oval circumscribed lesion on US. Readers 1 and 2 categorized this lesion as Breast Imaging Reporting and Data System (BI-RADS) category 4B, and reader 3 categorized this lesion as BI-RADS category 4A on DBT. Readers 2 and 3 categorized this lesion as BI-RADS category 3, and reader 1 categorized this lesion as BI-RADS category 4 A on US. 
Table 5. Summary of Malignant Cases Underestimated as Benign at Either DBT or US by at Least One Leader

\begin{tabular}{|c|c|c|c|c|c|c|}
\hline Case No. & Age (yr) & $\begin{array}{c}\text { Lesion } \\
\text { Size }(\mathrm{mm})\end{array}$ & $\begin{array}{c}\text { Underestimating } \\
\text { Modality }\end{array}$ & $\begin{array}{c}\text { BI-RADS Category } \\
\text { at DBT }\end{array}$ & $\begin{array}{c}\text { BI-RADS Category } \\
\text { at US }\end{array}$ & Pathology \\
\hline 1 & 62 & 12 & DBT & $\begin{array}{l}3 \text { (reader } 1,3) \\
4 \mathrm{~A}(\text { reader } 2)\end{array}$ & $\begin{array}{l}4 \mathrm{~A}(\text { reader } 3) \\
4 \mathrm{C}(\text { reader } 1,2)\end{array}$ & IDC \\
\hline 2 & 69 & 15 & DBT & $\begin{array}{c}3(\text { reader } 3) \\
4 B(\text { reader } 1,2)\end{array}$ & $\begin{array}{l}4 \mathrm{~B}(\text { reader } 1,2) \\
4 \mathrm{C}(\text { reader } 3)\end{array}$ & IDC with high grade DCIS \\
\hline 3 & 47 & 11 & DBT & $\begin{array}{c}3(\text { reader } 3) \\
4 B(\text { reader } 1,2)\end{array}$ & $\begin{array}{l}\text { 4A (reader } 3) \\
\text { 4B (reader } 2) \\
\text { 4C (reader } 1)\end{array}$ & Mucinous cancer with high grade DCIS \\
\hline 4 & 42 & 24 & DBT & $\begin{array}{c}3(\text { reader } 3) \\
4 B(\text { reader } 1,2)\end{array}$ & $\begin{array}{l}4 \mathrm{~B}(\text { reader } 2) \\
4 \mathrm{C}(\text { reader } 1,3)\end{array}$ & IDC \\
\hline 5 & 48 & 30 & DBT & $\begin{array}{r}3(\text { reader } 3) \\
4 \mathrm{~A}(\text { reader } 2) \\
4 \mathrm{~B}(\text { reader } 1)\end{array}$ & $4 \mathrm{~B}($ reader $1,2,3)$ & IDC \\
\hline 6 & 57 & 12 & US & $\begin{array}{l}4 \mathrm{~A}(\text { reader } 3) \\
4 \mathrm{~B}(\text { reader } 1,2)\end{array}$ & $\begin{array}{l}3 \text { (reader } 2,3) \\
4 \mathrm{~A}(\text { reader } 1)\end{array}$ & Invasive papillary carcinoma \\
\hline 7 & 38 & 10 & US & $\begin{array}{l}4 \mathrm{~A}(\text { reader } 1,3) \\
4 \mathrm{C}(\text { reader } 2)\end{array}$ & $\begin{array}{c}3 \text { (reader } 3) \\
4 \mathrm{~A}(\text { reader } 1,2)\end{array}$ & IDC with intermediate grade DCIS \\
\hline
\end{tabular}

Note.- BI-RADS = Breast Imaging Reporting and Data System, DBT = digital breast tomosynthesis, DCIS = ductal carcinoma in situ, IDC = infiltrating ductal carcinoma, US = ultrasound

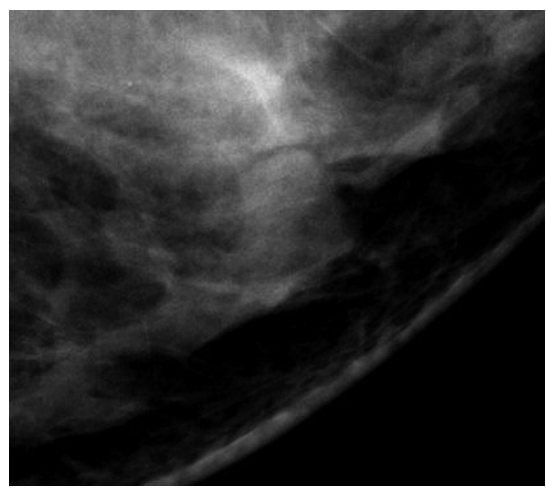

A
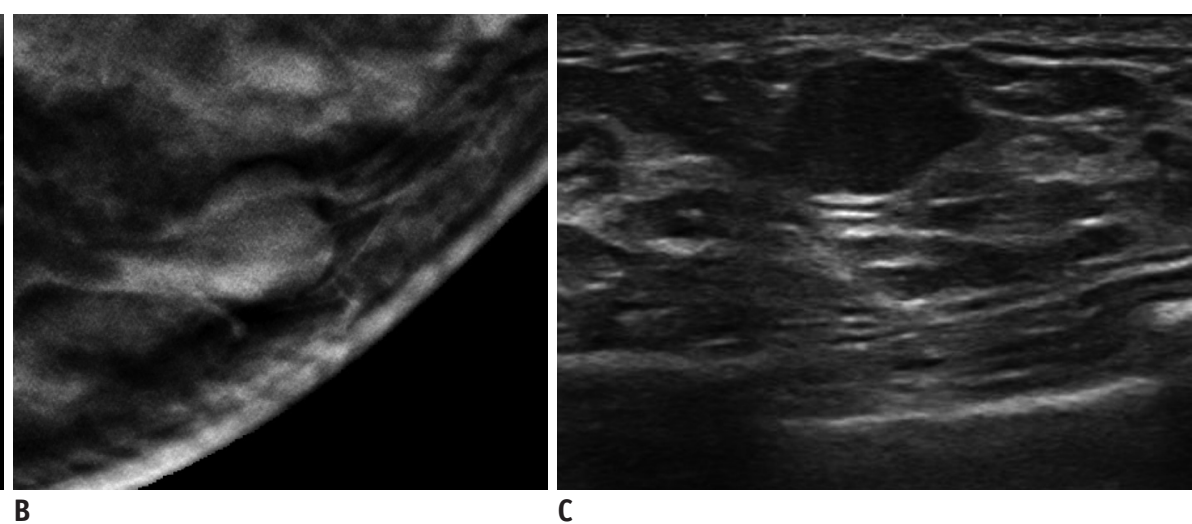

Fig. 3. 63-year-old woman with fibroadenoma $(1.3 \mathrm{~cm}$ in diameter as measured on ultrasonography [US]).

A. Mediolateral oblique digital mammography view. B. Mediolateral oblique digital breast tomosynthesis (DBT) (1-mm section) view. C. Transverse US view. Round isodense mass with circumscribed margins is observed on DBT. This lesion appears as irregular hypoechoic circumscribed mass on US. Readers 1 and 2 categorized this lesion as Breast Imaging Reporting and Data System (BI-RADS) category 3, and reader 3 categorized this lesion as BI-RADS category 2 on DBT. Readers 1 and 2 categorized this lesion as BI-RADS category 4A, and reader 3 categorized this lesion as BIRADS category 3 on US.

presence of calcifications (0.611), moderate for margins (0.414), orientation (0.593), lesion boundaries (0.498), and posterior acoustic features (0.471); and fair for the echo pattern (0.344). The interobserver agreement among the three readers for the final BI-RADS assessment of the breast masses was similar for DBT (value, 0.545; standard error, 0.024) and US (value, 0.602; standard error, 0.026).

\section{DISCUSSION}

Our results indicate that the diagnostic performance for characterizing lesions was comparable between DBT and US. The overall AUC values and mean sensitivities and specificities were not different for the breast lesions presented as abnormalities on routine mammography.

Many researchers have investigated the potential role of DBT in both screening and diagnostic settings. Improvements in sensitivity and specificity are expected after adding DBT to conventional mammography because DBT eliminates overlapping tissues, and lesion margins can be more readily assessed, which may reduce the need for extra views $(15,25)$. In a recent study by Zuley et al. 


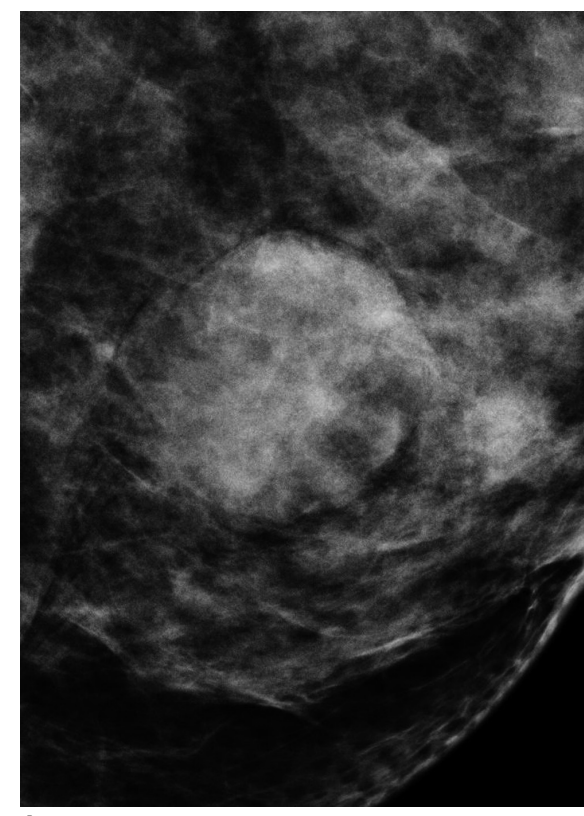

A

Fig. 4. 38-year-old woman with $2.5-\mathrm{cm}$ benign phyllodes tumor.

A. Mediolateral oblique digital mammography view. B. Mediolateral oblique digital breast tomosynthesis (DBT) (1-mm section) view. C. Transverse ultrasonography (US) view. Circumscribed high density mass is observed on DBT images. Mass is oval, circumscribed and accompanied by posterior acoustic enhancement on US. All readers categorized this lesion as Breast Imaging Reporting and Data System (BI-RADS) category 4A on DBT. All readers categorized this lesion as BI-RADS category 3 on US.

(26), DBT significantly improved diagnostic accuracy for noncalcified lesions compared with that of supplemental mammographic views. Hence, DBT could be a possible alternative for additional mammographic views which has been performed for a long time to characterize lesions.

Breast US has been used to evaluate specific areas of abnormality discovered on either a clinical examination or mammography. Previous prospective clinical studies have demonstrated that appropriate use of US as an adjunct to mammography improves sensitivity and specificity of breast cancer diagnoses $(27,28)$, particularly in women with dense breasts and in younger women $(22,29)$.

The average sensitivities of both DBT and US were > 95\% (US range, $97.3-100 \%$ ) using BI-RADS category 4 as a cut-off point, whereas the average false-positive rates of DBT and US were approximately 55\% (range, 27.3-75\%) and $60 \%$ (range, 56.8-68.2\%), respectively. Only a few studies have discussed the diagnostic performance of DBT vs. US $(16,29,30)$. No significant difference in overall diagnostic performance (lesion differentiation) was found between single-view DBT and dual-view mammography with US in a side-by-side review of 40 cancers with subtle signs on mammography (30). Mammography combined with US missed two cancers detected by DBT, whereas it revealed seven other cancers missed by DBT. These findings are similar to our results, in which five cancers that were considered benign on DBT were correctly categorized as malignant on US, and two cancers categorized as benign on US images were correctly categorized as malignant on DBT images. In addition, Hakim et al. (16) also reported that the availability of DBT would have eliminated the need for US as a part of the diagnostic process in $32 \%$ of their cases. Thus, our findings of comparable diagnostic performances hint that DBT could be utilized instead of US in to characterize lesions visible on MG in a diagnostic setting, even though it may not be superior to US for detecting cancer in a screening setting. Our interobserver agreement results were similar to a previous report (31), and considering that reader experience with US was consistently longer than that of DBT, our results could be encouraging evidence for DBT to be used to characterize lesions instead of US.

In our study, DBT and US specificities were lower than those of many other studies $(13,22)$, and $20.5-59.1 \%$ of benign lesions were recommended for biopsy in both imaging tests by the three readers, which may have occurred because our study population was composed of patients with mammographically abnormal findings, our study population was enriched, and the readers tended to assess the lesions more suspiciously than in a routine 
clinical setting.

Our study had several limitations. First, our study was a retrospective review, and only static B-mode US images were used for the reader study; therefore, the results may not completely translate to the clinical environment. Elastography and Doppler images provide more detailed information for lesion assessment; thus, improved diagnostic performance could be achieved (32). Second, the study population was enriched with malignant lesions, and the number of lesions was small. The type of lesion (benign vs. malignant and cyst vs. solid) and patient characteristics can also affect DBT and US diagnostic performance. Only a small portion of subjects in a breast cancer screening program have malignant lesions, and the majority of subjects have benign lesions, such as cysts and fibroadenomas. We may have reached a different conclusion with a screening population. Third, the number of benign cysts was small. DBT probably cannot distinguish whether a well-defined mass is cystic or solid, or complex cystic, but US differentiate these without difficulties. Fourth, all three readers had more experience interpreting US images than those of DBT. This difference in experience may have been the reason for the relatively low interobserver agreement values. In addition, diagnostic performance according to reader experience was not assessed. Fifth, the reader study was done without knowledge of MG findings. This is different from the initial state of US image acquisition; thus, producing different results. Lastly, most of the masses had been detected on conventional DM as part of standard screening or a diagnostic evaluation, thereby biasing the study against DBT and US because those cancers that could only be detected by DBT or US were not included. However, we focused only on characterizing mammographically visible lesions, and our results provide guidance in a similar clinical setting.

In conclusion, DBT offered similar diagnostic performance compared to that of US for characterizing breast lesions depicted on mammography. DBT may be useful to characterize lesions with similar performance to US when mammographically visible lesions are detected.

\section{REFERENCES}

1. Berry DA, Cronin KA, Plevritis SK, Fryback DG, Clarke L, Zelen $M$, et al. Effect of screening and adjuvant therapy on mortality from breast cancer. N Engl J Med 2005;353:17841792

2. Hellquist BN, Duffy SW, Abdsaleh S, Björneld L, Bordás $P$,
Tabár $L$, et al. Effectiveness of population-based service screening with mammography for women ages 40 to 49 years: evaluation of the Swedish Mammography Screening in Young Women (SCRY) cohort. Cancer 2011;117:714-722

3. Tabár L, Fagerberg CJ, Gad A, Baldetorp L, Holmberg LH, Gröntoft 0 , et al. Reduction in mortality from breast cancer after mass screening with mammography. Randomised trial from the Breast Cancer Screening Working Group of the Swedish National Board of Health and Welfare. Lancet 1985;1:829-832

4. Tabar L, Yen MF, Vitak B, Chen HH, Smith RA, Duffy SW. Mammography service screening and mortality in breast cancer patients: 20-year follow-up before and after introduction of screening. Lancet 2003;361:1405-1410

5. Mandelson MT, Oestreicher N, Porter PL, White D, Finder $\mathrm{CA}$, Taplin $\mathrm{SH}$, et al. Breast density as a predictor of mammographic detection: comparison of interval- and screendetected cancers. J Natl Cancer Inst 2000;92:1081-1087

6. Kerlikowske K, Grady D, Barclay J, Sickles EA, Ernster V. Effect of age, breast density, and family history on the sensitivity of first screening mammography. JAMA 1996;276:33-38

7. Kopans DB. Digital breast tomosynthesis from concept to clinical care. AJR Am J Roentgenol 2014;202:299-308

8. Niklason LT, Christian BT, Niklason LE, Kopans DB, Castleberry $\mathrm{DE}$, Opsahl-Ong BH, et al. Digital tomosynthesis in breast imaging. Radiology 1997;205:399-406

9. Gennaro G, Toledano A, di Maggio C, Baldan E, Bezzon E, La Grassa M, et al. Digital breast tomosynthesis versus digital mammography: a clinical performance study. Eur Radiol 2010;20:1545-1553

10. Good WF, Abrams GS, Catullo VJ, Chough DM, Ganott MA, Hakim CM, et al. Digital breast tomosynthesis: a pilot observer study. AJR Am J Roentgenol 2008;190:865-869

11. Gur D, Abrams GS, Chough DM, Ganott MA, Hakim CM, Perrin $\mathrm{RL}$, et al. Digital breast tomosynthesis: observer performance study. AJR Am J Roentgenol 2009;193:586-591

12. Spangler ML, Zuley ML, Sumkin JH, Abrams G, Ganott MA, Hakim C, et al. Detection and classification of calcifications on digital breast tomosynthesis and 2D digital mammography: a comparison. AJR Am J Roentgenol 2011;196:320-324

13. Tagliafico A, Astengo D, Cavagnetto F, Rosasco R, Rescinito G, Monetti $F$, et al. One-to-one comparison between digital spot compression view and digital breast tomosynthesis. Eur Radiol 2012;22:539-544

14. Teertstra HJ, Loo CE, van den Bosch MA, van Tinteren $H$, Rutgers EJ, Muller SH, et al. Breast tomosynthesis in clinical practice: initial results. Eur Radiol 2010;20:16-24

15. Noroozian M, Hadjiiski L, Rahnama-Moghadam S, Klein KA, Jeffries D0, Pinsky RW, et al. Digital breast tomosynthesis is comparable to mammographic spot views for mass characterization. Radiology 2012;262:61-68

16. Hakim CM, Chough DM, Ganott MA, Sumkin JH, Zuley ML, Gur D. Digital breast tomosynthesis in the diagnostic environment: a subjective side-by-side review. AJR Am J Roentgenol 2010;195:W172-W176 
17. Frazier TG, Murphy JT, Furlong A. The selected use of ultrasound mammography to improve diagnostic accuracy in carcinoma of the breast. J Surg Oncol 1985;29:231-232

18. Kaplan SS. Clinical utility of bilateral whole-breast US in the evaluation of women with dense breast tissue. Radiology 2001;221:641-649

19. Bassett LW. Imaging of breast masses. Radiol Clin North Am 2000;38:669-691, vii-viii

20. Dennis MA, Parker SH, Klaus AJ, Stavros AT, Kaske TI, Clark SB. Breast biopsy avoidance: the value of normal mammograms and normal sonograms in the setting of a palpable lump. Radiology 2001;219:186-191

21. Graf 0, Helbich TH, Hopf G, Graf C, Sickles EA. Probably benign breast masses at US: is follow-up an acceptable alternative to biopsy? Radiology 2007;244:87-93

22. Houssami N, Irwig L, Simpson JM, McKessar M, Blome S, Noakes J. Sydney Breast Imaging Accuracy Study: comparative sensitivity and specificity of mammography and sonography in young women with symptoms. AJR Am J Roentgenol 2003;180:935-940

23. D'Orsi CJ, Bassett LW, Berg WA, Feig SA, Jackson VP, Kopans DB, et al. Breast Imaging Reporting and Data System, BIRADS: Mammography, 4th ed. Reston: American College of Radiology, 2003

24. Mendelson EB, Baum JK, Berg WA, Merritt CB, Rubin E. Breast Imaging Reporting and Data System, BI-RADS: Ultrasound, 1st ed. Reston: American College of Radiology, 2003

25. Rafferty EA, Park JM, Philpotts LE, Poplack SP, Sumkin JH, Halpern $E F$, et al. Assessing radiologist performance using combined digital mammography and breast tomosynthesis compared with digital mammography alone: results of a multicenter, multireader trial. Radiology 2013;266:104-113

26. Zuley ML, Bandos AI, Ganott MA, Sumkin JH, Kelly AE, Catullo VJ, et al. Digital breast tomosynthesis versus supplemental diagnostic mammographic views for evaluation of noncalcified breast lesions. Radiology 2013;266:89-95

27. Vercauteren LD, Kessels AG, van der Weijden T, Koster D, Severens JL, van Engelshoven JM, et al. Clinical impact of the use of additional ultrasonography in diagnostic breast imaging. Eur Radiol 2008;18:2076-2084

28. Flobbe K, Bosch AM, Kessels AG, Beets GL, Nelemans PJ, von Meyenfeldt MF, et al. The additional diagnostic value of ultrasonography in the diagnosis of breast cancer. Arch Intern Med 2003;163:1194-1199

29. Leconte I, Feger C, Galant C, Berlière M, Berg BV, D'Hoore W, et al. Mammography and subsequent whole-breast sonography of nonpalpable breast cancers: the importance of radiologic breast density. AJR Am J Roentgenol 2003;180:1675-1679

30. Andersson I, Ikeda DM, Zackrisson S, Ruschin M, Svahn T, Timberg $P$, et al. Breast tomosynthesis and digital mammography: a comparison of breast cancer visibility and BIRADS classification in a population of cancers with subtle mammographic findings. Eur Radiol 2008;18:2817-2825

31. Lazarus E, Mainiero MB, Schepps B, Koelliker SL, Livingston LS. BI-RADS lexicon for US and mammography: interobserver variability and positive predictive value. Radiology 2006;239:385-391

32. Lee SH, Chang JM, Cho N, Koo HR, Yi A, Kim SJ, et al. Practice guideline for the performance of breast ultrasound elastography. Ultrasonography 2014;33:3-10 